International Journal of Advanced Multidisciplinary Research

ISSN: 2393-8870

www.ijarm.com

(A Peer Reviewed, Referred, Indexed and Open Access Journal)

DOI: 10.22192/ijamr

Volume 8, Issue 6 -2021

Review Article

DOI: http://dx.doi.org/10.22192/ijamr.2021.08.06.006

\title{
TNBC: A brief discussion on Triple Negative Breast Cancer
}

\section{Dipty Tandi}

M. Sc Microbiology

Mody University of Science \& Technology, Sikar, Rajasthan

E-mail: diptytandi21@gmail.com

Keywords

TNBC;

female hormone,

breast cancer,

BRCA group.
Abstract

TNBC; Triple negative breast cancer is one of the most aggressive type of breast cancer with a high reoccurrence and mortality rate. The reason behind its aggressive type is that TNBC do not have such specific receptors as other cancers. Substantially, Breast tumor cells carries receptors that work as doors for many mechanisms and hormone/enzyme based treatment. These receptors are estrogen (female hormone), Progesterone (female hormone) and HER-2 (Protein; Human epidermal receptor). Absence of these enzymes is result into the less option availability in cancer treatment. To find more possible treatments like chemotherapy, lumpectomy, radiation therapy and other gene therapy we first need to understand the concept behind occurrence and reoccurrence of TNBC. This review listed the possible study of triple negative breast cancer including its appearance in society and the molecular/genetic involvement. It is formerly known that cancer take place due to mutation in BRCA group of genes so it is important to know how it works in the case of TNBC. This study also summarizes the influence of anti-diabetic drug treatment (e.g. metformin) on diabetic cancer patients in order to cancer therapy of TNBC and effect of high glucose concentric cells on anticancer drug treatments and strategies for development in TNBC development. 


\section{Introduction}

Triple negative breast cancer is termed triple negative due to its characteristic features as it does not express much of HER2 (neu-gene) (human epidermal receptor-2) and there is absence of progesterone and estrogen receptors, whereas in the other types of breast cancer either one or two of them are usually present to help in growth of tumor cells [1,2]. TNBC cases are commonly at higher risk of metastasis to lung and brain and reoccur more early as compare to other sub-categories of breast cancer $[7,8]$. TNBC is an aggressive breast cancer, according to mitotic index of pathologic features; it is on a higher histologic grade [9]. TNBC has higher rate of LRR (local recurrence rate), and poor CSS (cancer-specific survival) and DFS (disease-free survival) (6).

Claudin low breast cancer and Basal like breast cancer should always be mention while TNBC is described because molecular pathology and profiling of gene expression shows that breast cancer is divided into following subtypes, namely lumina A, lumina B, claudin, basal like and HER2 enriched breast cancer (2). Characteristically, Luminal A BC possesses Higher expression of estrogen receptors (ER) and ER-linked genes, Luminal B associate with higher rate of tumor proliferation and express lower level of ER and associated genes, HER2 enriched express higher level of both GRB7, it demonstrate the reduced upshot of HER2 targeted agent. Basal like BC expresses basal cytokeratin positively and expresses HER2 and luminal genes negatively. Moreover, at the gene level basal-like BC and HER2 BC characteristically shows the breast basal epithelial cells (3). Some data shows that majority of TNBC cases are of basal like breast cancer cases. The claudin-low Breast Cancer is subjected to less expression of claudin proteins, which are involved in cell-cell adhesion. Consciously, higher level expression of putative stem cell marker and EMT are negetively associated with HER2 and ER but in basal cytokeratin its expression is low. For the research most likely used method is micro-array gene expression assay.

\section{Role of BRCA genes in TNBC}

TNBC is not the normal type of breast cancer as it does not contain its specific receptor which makes it more hazardous because absence of such receptor came with the disadvantages that it cannot be treated with enzymes/hormone based treatment. Weather the positive fact is hormonal or enzymatic activities cannot induce its affectivity. Normal cells become cancer cells due to changes or mutations in the DNA. While people inherit some DNA changes, others acquire these DNA changes during a person's life. Generally tnbc is being developed due to unhealthy lifestyle changes, obesity and inheritable reasons. Development of metastases in Triple Negative Breast Cancer (TBNC) undergoes many different pathways including epigenetic/genetic alteration, tumor stroma exchanges, angiogenesis, intravasion in base membrane, extravasation in distal tissue and circulation survival (Neophytou et al., 2018).

According to some researches it is possibly said that BRCA1/2 mutation is one of the main cause of many cancers including Triple-Negative-Breast cancer/TNBC. BRCA1 and BRCA2 are the genes of normal cells if they function as they are supposed to do it helps to prevent from cancer but some unwanted mutation in these genes resultant in defective protein and causes cancerous or tumor cells to be developed. These mutations make the other cell susceptible for other genetic alteration that give rise to cancer. TripleNegatice-Breast-Cancer is the most likely occurring cancer due to BRCA1 mutation. BRCA $1 / 2$ protein plays a major character in DNA repair, homologous recombination and stabilization of DNA replication fork. It is also a part of protein network in fanconi anemia (Tutt et al., 2018). Functions of BRCA 1 and BRCA2 are collectively called BRCAness.

BRCA1 and BRCA2 germline mutation can cause breast cancer in individual. This mutation occurs due to replication stress and abnormal DNA repair. These abnormalities take places mainly during Methylation in promoter of 
BRCA1-mRNA. This cancer is also called gBRCA-BC (germline mutated breast cancer) by this homologous recombination, genomic instability take place. Homologous recombination also repairs the DNA damage caused by platinum agents (Tutt et al., 2018). There is also a hypothesis that in some population of Triplenegative breast cancers are having platinum sensitive BRCA1/BRCA2 mutation (Tutt et al., 2018). Some mutational homologous recombination deficiency and low leveled BRCA1 mutations are also platinum sensitive. Analysis of genetic comparison between BRCA1/2 doesn't make any difference also it is reported that in such cases with BRCA1 and 2 mutations there was very less number of ER positive tumors. It means those genes do not influence one another's affectivity. Epigenetic analysis of gene silencing showed that low mRNA tumors or BRCA1 methylation is more sensitive toward docetaxel rather than carboplatin. This silencing show that in DNA Methylation, BRCA1 mutation shows similar treatment in subject to epigenetic analysis of BRCAness. Responses of and toward this methylation is been recorded as genetical biomarkers of BRCA1/2 mutation. All these perspectives and detections are used to treat this BRCA mutated TNBC (Triple-egetive-breast-cancer). These reports are also useful to design more appropriate chemotherapy (platinum chemotherapy) as TNBC is a cancer with very high mortality rate.

Examinations of paired initial and metastatic TNBC samples are required for better understanding of disease reoccurrence. In Most cases of Triple negative breast cancer Metastases and primary tumors both are polyclonal and associated with overlapped clones, showing that polyclonal metastases are frequent in TNBC. With the help of Phylogenetic analysis it is easy to distinguish between local reoccurrence by $2^{\text {nd }}$ primary tumor also it is helpful to determine the source of metastasis in patient with previous tumor history (Yates et al., 2017). Understanding the cause and origin of primary and reoccurred metastasis can affect the strategies for management of cancer and future treatment aspects (Garrido-Castro et al., 2019).

\section{Clinical features of TNBC}

TNBC tumor is one of most aggressive tumor known. Its pathologic features include elevated mean tumor size, high rate of node positivity, incept at younger ages, higher mitotic index and higher morality (Andres et al., 2008; Moah et al., 2020). Triple negative breast cancer is a heterogeneous type of breast cancer. Instinctive metastatis is more common in TNBC as compare to the ER-positive cancers (Tan et al., 2018). Its distant disease site is mainly place in lungs and CNS (central nervous system) (Tan et al., 2018). Subsequent to neoadjuvant chemotherapy TNBC respond better to the cytotoxic chemotherapy in order to higher rate of pathologic response. But tnbc has inferior prognosis in comparison of other breast cancers this phenomenon is termed as a paradox of triple negative breast cancer (Tan et al., 2018). Other risk factors of TNBC include obesity, duration of breastfeeding and parity. As TNBC is different from other subtypes of breast cancer its risk factor is also differ from others. In normal case of positive receptor breast cancer high parity, long duration of breastfeeding and reduced exposure of estrogen saves from cancer severity but in triple negative breast cancer high parity, younger age pregnancy and overweight females are at higher risk (Tan et al., 2018).

It is reported that TNBC mainly occurs in younger females before menopause (CDC report 2020). Risk of TNBC varies with the people of different region and races. According to a population based study of TNBC cancer with molecular and environmental perspective It has been perceived that black people or Africans has more risk of death with TNBC while there is higher chance of TNBC occurrence in white people but low mortality rate (according to CDC report) (Tan et al., 2018). TNBC subtype of basal tumor like cancer played an important role in this study. It is rectified by immunohistochemical markers such as cytokeratin 5/6+, HER1+, ERand HER2-. 


\section{Molecular and genetic analysis of TNBC}

TNBC is subdivided in groups on the basis of histological, clinical and molecular subtyping (Dong et al., 2018) (Peddi et al., 2012). All these subtypes are identified with the help of genomic DNA micro array, m-RNA array, DNA Methylation, coding DNA sequencing, protein array and mi-RNA sequencing methods and techniques (Dong et al., 2018). These studies were also helpful in intensive understanding of molecular pattern of triple negative breast cancer that helps to find the novel approach for its treatment (Dong et al., 2018).

There are five subtypes of TNBC on the basis of molecular pattern subtyping. That is luminal A, luminal B, Basal like, HER-2 enriched, normallike and claudin low subtype of TNBC (Peddi et al., 2012). Trancriptomic analysis TNBC heterogeneity identifies following subtypes according to specific gene profiling BL1 subtype (basal like-1), BL2 subtype (basal like-2), M subtype (mesenchymal), MSL subtype (mesenchymal stem like), IM subtype (Immunomodulatory), LAR subtype (luminal-androgenreceptor) and UNS (Unstable-cluster) (GarridoCastro et al., 2019). This classification characterized on the basis of their distinct molecular pattern, RNA sequencing, gene implication in various pathways and mutation in somatic cell (Garrido-Castro et al., 2019).

\begin{tabular}{|c|c|c|}
\hline Sr.No. & $\begin{array}{l}\text { Subtypes of breast cancer } \\
\quad \text { (Intrinsic subtypes) }\end{array}$ & $\begin{array}{c}\text { Subtypes of TNBC } \\
\text { (On the basis of gene expression profiling }\end{array}$ \\
\hline 1. & Luminal A & $\begin{array}{l}\text { BL-1 } \\
\text { (basal-like) }\end{array}$ \\
\hline 2. & Luminal -B & $\begin{array}{l}\text { BL-2 } \\
\text { (basal-like) }\end{array}$ \\
\hline 3. & Basal-like & $\begin{array}{l}\text { IM } \\
\text { (immunomodulatory) }\end{array}$ \\
\hline 4. & HER-2 Enriched & M (Mesenchymal) \\
\hline 5 . & Normal-like & $\begin{array}{l}\text { MSL } \\
\text { (Mesenchymal stem like) }\end{array}$ \\
\hline 6. & Claudin-low & $\begin{array}{l}\text { LAR } \\
\text { (Luminal androgen receptor) }\end{array}$ \\
\hline
\end{tabular}

Currently DEG genes (differentially expressed genes) are identified by the gene expression profiling comparison between TNBC positive and TNBC negative patients. Then it underwent various analysis such as GO; gene ontology, PPI; protein protein interaction and KEGG; kyto encyclopedia of gene-genome pathway (Yin et al., 2020). These examinations may open the door for future advancement in TNBC prognosis, diagnosis, drug discovery and targeted therapy (Yin et al., 2020).

Epigenetic markers can play a major role in treatment and diagnose of triple negative breast cancer. Because an epigenetic alteration is change in promoter gene DNA Methylation and post translational modification in Histone proteins that influence gene expression (Garrido-Castro et al., 2019). These changes/ alteration are recognizable characteristic of TNBC. TNBC comprehensively demonstrate the $\mathrm{CpG}$ Methylation of promoter regions of 9 following epigenetic marker genesCEACAM6, CDH1, CST6, ESR1, GNA11, MUC1, SCNN1A, MYB and TFF3 (GarridoCastro et al., 2019). Novel strategies in epigenetic modification incorporate BET bromodomain inhibitors which bind with acetylated lysine residues of Histone proteins that promote displacement of bromodomain from chromatin and disrupt the transcriptional activity (Belkina et al., 2012). BET inhibitors attain persuasive repression of tumor growth in TNBCs cell lines 
exemplified by more basal-like, claudin-low and/or stem cell like characteristics (Shu et al., 2016).

\section{Influence of obesity and Diabetes on TNBC}

Obesity and diabetes/metabolic syndrome are always a risk factor in every disease. This is also attributed that mechanism that promotes TNBC is conducted by obesity in such way of metamorphosis of testosterone in adipocytes. It leads to elevated circulation of bio accessible oestrogen that can cause problem in postmenopausal stages (vecchia et al., 2011). Current data of breast cancer suggest that obesity promotes tumor progression by many mechanisms, for example shift in fat and carbohydrate metabolism, regulation of carcinogens that support the tumor growth (e.g. cytokines, IL-growth facors, insulin), macrophage activation and immune response activation etc. (Verghese et al., 2019).

Characteristically high blood sugar level raises the risk of death in TNBC patient. But such antidiabetic drug treatments as Metformin verified to be helpful in TNBC therapy as it reduces the proliferation, inhibit the mTOR pathway and improves the survival rate (Verghese et al., 2019). mTOR pathway is a pathway for cell-signaling that place a central role in proliferation and cell growth which promotes tumor formation. High concentration of glucose in cells elevates the risk of Triple Negative breast cancer. Metformin is a most commonly used anti-diabetic drug that lowers the risk of TNBC in order of its mechanism of action against lipid metabolism shift (welhard-alaska et al., 2014) (Verghese et al., 2019).

As we stated earlier, higher glucose level promotes cancer aggression but metformins mechanism of action differ by molecular subtypes of disease and that type of triple negative breast cancer are usually sensitive towards its anticancer properties (wahdan-Alaswad et al., 2013) . Moreover, metformin inhibits cell proliferation, decreases oncogenecity, lowers the motility, target stem cells and promotes apoptosis (liu et al., 2011). By the recent experiments it is observed that Metformin on breast tumor take place more in obese as compare to lean mice and in overfed obese mice. Reports from these models are suggesting that metabolic deregulation is linked with obesity on breast tumor progression, development and efficacy of Metformin (wahdan et al., 2013; liu et al., 2011).

Metformin; an anti-diabetic drug, minimizes the insulin resistance and induces lipid and glucose homeostatis mainly in skeletan and liver muscles. The broadly significant mechanism of action of Metformin by inhibiting targets complex of rapamycin (mTORC1) in both pathways of AMPK (AMPK-dependent \& AMPKindependent) (Deng et al., 2012). The effect of Metformin on benign and malignant cancer is still not properly known. Metformin attenuate various tumor formation or tumor progression signaling pathways such as STAT3, OCT1 (organic +ion transporter) and TGF- $\beta$ (transforming growth factor). These mechanisms as drug effect of Metformin on cancer cells can be very much help for approaching new therapies of triple negative breast cancer.

\section{Future aspects for Strategies to treat TNBC}

Most commonly used treatments for TNBC and other cancers are chemotherapy, immunotherapy and other targeted therapies that improve the survival rate for patients (Bath et al., 2019). Rather than that standard neoadjuvant therapy is proposed in which taxane-anthracycline based chemotherapy is used. In phase-II trial 1-SPY-2 was randomly used and evaluated the inhibitor namely pembrolizumab with standard taxane subsequent to the anthramycin dependent chemotherapy. This strategy was found to be very much vigorous in the case if triple negative breast cancer and also in the patient with positive hormone receptor disease (Bath et al., 2019). This result also suggest that toxicity of immunotherapeutic drugs should be monitored even after the completion the therapy that should keep examined for some time for post toxic effects or long term outcome. 
Anti-body drug conjugates has been shown as new effective anti-cancer drug. The concept behind this is, an antibody which is extremely selective for tumor linked antigens because it won't be expressed in normal or healthy cells. An example of anti-body drug conjugate is T-DM1 (trastuzumab emtansine) it is approved for HER-2 positive breast cancer and others are in the process of development. These discoveries are very much helpful for development of new drugs in TNBC case for its management (Rugo et al., 2019)

\section{References}

1. Belkina AC, Denis GV. BET domain coregulators in obesity, inflammation and cancer. Nat Rev Cancer 2012; 12:465-77.

2. Checkley LA, Rudolph MC, Wellberg EA, Giles ED, Wahdan-Alaswad RS, et al. Metformin Accumulation Correlates with Organic Cation Transporter 2 Protein Expression and Predicts Mammary Tumor Regression In Vivo. Cancer Prev Res (Phila) 2017; 10:198-207.

3. Deng XS, Wang S, Deng A, Liu B, Edgerton SM, et al. Metformin targets Stat3 to inhibit cell growth and induce apoptosis in triple-negative breast cancers. Cell Cycle. 2012; 11:367-376.

4. Deng, Xin-Sheng \& Wang, Shuiliang \& Deng, Anlong \& Liu, Bolin \& Edgerton, Susan \& Lind, Stuart \& Wahdan-Alaswad, Reema \& Thor, Ann. (2012). Metformin targets Stat3 to inhibit cell growth and induce apoptosis in triple-negative breast cancers. Cell cycle (Georgetown, Tex.). 11. 367-76. 10.4161/cc.11.2.18813.

5. E. A. Rakha, S. E. Elsheikh, M. A. Aleskandarany et al., "Triple-negative breast cancer: distinguishing between basal and nonbasal subtypes," Clinical Cancer Research, vol. 15, no. 7, pp. 2302-2310, 2009.
6. F. Bertucci, P. Finetti, N. Cervera et al., "How basal are triplenegative breast cancers?" International Journal of Cancer, vol. 123, no. 1, pp. 236-240, 2008.

7. Fabio Puglisi, Caterina Fontanella, Vito Amoroso, Giulia Valeria Bianchi, Giancarlo Bisagni, Cristina Falci, Andrea Fontana, Daniele Generali, Lorenzo Gianni, Antonio Grassadonia, Luca Moscetti, Ilaria Portarena, Emanuela Rossi, Paolo Marchetti. Current challenges in HER2positive breast cancer, Critical Reviews in Oncology/Hematology. Vol. 98 2016. 211221 ISSN $1040-8428$ https://doi.org/10.1016/j.critrevonc.2015.10. $\underline{016}$

8. Feng T, Cao W, Shen W, et al. Arctigenin inhibits STAT3 and exhibits anticancer potential in human triple-negative breast cancer therapy. Oncotarget. 2017;8(1):329344. doi:10.18632/oncotarget.13393

9. Garrido-Castro AC, Lin NU, Polyak K. Insights into Molecular Classifications of Triple-Negative Breast Cancer: Improving Patient Selection for Treatment. Cancer Discov. 2019 Feb;9(2):176-198. doi: 10.1158/2159-8290.CD-18-1177. Epub 2019 Jan 24. PMID: 30679171; PMCID: PMC6387871.

10. Knight JF, Lesurf R, Zhao H, et al. Met synergizes with p53 loss to induce mammary tumors that possess features of claudin-low breast cancer. Proc Natl Acad Sci U S A 2013; 110:E1301-10.

11. La Vecchia C, Giordano SH, Hortobagyi GN, Chabner B. Overweight, obesity, diabetes, and risk of breast cancer: interlocking pieces of the puzzle. Oncologist. 2011; 16:726-729.

12. Liu B, Fan Z, Edgerton SM, Yang X, Lind SE, Thor AD. Potent antiproliferative effects of metformin on trastuzumabresistant breast cancer cells via inhibition of erbB2/IGF-1 receptor interactions. Cell Cycle. 2011; 10:2959-2966. 
13. Metformin-induced killing of triplenegative breast cancer cells is mediated by reduction in fatty acid synthase via miRNA193b. Wahdan-Alaswad RS, Cochrane DR, Spoelstra NS, Howe EN, Edgerton SM, Anderson SM, Thor AD, Richer JK Horm Cancer. 2014 Dec; 5(6):374-89.

14. Neophytou C, Boutsikos P, Papageorgis P. Molecular Mechanisms and Emerging Therapeutic Targets of Triple-Negative Breast Cancer Metastasis. Front Oncol. 2018 Feb 22; 8:31. doi: 10.3389/fonc.2018.00031. PMID: 29520340 ; PMCID: PMC5827095.

15. Perou CM, Sørlie T, Eisen MB, et al. Molecular portraits of human breast tumours. Nature 2000; 406:747-52.

16. Prat A, Parker JS, Karginova O, et al. Phenotypic and molecular characterization of the claudin-low intrinsic subtype of breast cancer. Breast Cancer Res 2010; 12:R68.

17. R. Dent, M. Trudeau, K. I. Pritchard et al., "Triple-negative breast cancer: clinical features and patterns of recurrence," Clinical Cancer Research, vol. 13, no. 15, pp. 4429-4434, 2007.

18. R. Dent, W. M. Hanna, M. Trudeau, E. Rawlinson, P. Sun, and S. A. Narod, "Pattern of metastatic spread in triplenegative breast cancer," Breast Cancer Research and Treatment, vol. 115, no. 2, pp. 423-428, 2009.

19. Richardson LC, Henley J, Miller J, Massetti G, Thomas CC. Patterns and trends in black-white differences in breast cancer incidence and mortality-United States, 1999-2013. MMWR 2016; 65(40):10931098.
20. Rugo H: New options for patients with triple negative breast cancer. 2019 Lynn Sage Breast Cancer Symposium. Presented October 5, 2019.

21. Shu S, Lin CY, He HH, Witwicki RM, Tabassum DP, Roberts JM, et al. Response and resistance to BET bromodomain inhibitors in triple-negative breast cancer. Nature 2016; 529:413-7.

22. T. Sørlie, C. M. Perou, R. Tibshirani et al., "Gene expression patterns of breast carcinomas distinguish tumor subclasses with clinical implications," Proceedings of the National Academy of Sciences of the United States of America, vol. 98, no. 19, pp. 10869-10874, 2001.

23. Tutt, A., Tovey, H., Cheang, M.C.U. et al. Carboplatin in BRCA1/2-mutated and triple-negative breast cancer BRCAness subgroups: the TNT Trial. Nat Med 24, 628-637 (2018). https://doi.org/10.1038/s41591-018-0009-7

24. Wahdan-Alaswad R, Fan Z, Edgerton SM, Liu B, Deng XS, et al. Glucose promotes breast cancer aggression and reduces metformin efficacy. Cell Cycle. 2013;12:3759-3769

25. Wahdan-Alaswad R, Harrell JC, Fan Z, Edgerton SM, Liu B, et al. Metformin attenuates transforming growth factor beta (TGF-beta) mediated oncogenesis in mesenchymal stem-like/claudin-low triple negative breast cancer. Cell Cycle. 2016; 15:1046-1059.

26. Yates LR, Knappskog S, Wedge D, Farmer y JHR, Gonzalez S, Martincorena I, et al. Genomic evolution of breast cancer metastasis and relapse. Cancer Cell 2017; 32:169-84e7. 
27. Yin, L., Duan, J., Bian, X. et al. Triplenegative breast cancer molecular subtyping and treatment progress. Breast Cancer Res 22, 61 (2020).

https://doi.org/10.1186/s13058-020-01296-5

\begin{tabular}{|c|c|}
\hline \multicolumn{2}{|c|}{ Access this Article in Online } \\
\hline \multirow{2}{*}{ 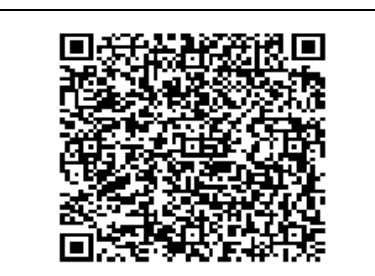 } & $\begin{array}{l}\text { Website: } \\
\text { www.ijarm.com }\end{array}$ \\
\hline & \multirow[t]{2}{*}{$\begin{array}{l}\text { Subject: } \\
\text { Oncology }\end{array}$} \\
\hline Quick Response Code & \\
\hline \multicolumn{2}{|c|}{ DOI:10.22192/ijamr.2021.08.06.006 } \\
\hline
\end{tabular}

How to cite this article:

Dipty Tandi. (2021). TNBC: A brief discussion on Triple Negative Breast Cancer. Int. J. Adv. Multidiscip. Res. 8(6): 75-82.

DOI: http://dx.doi.org/10.22192/ijamr.2021.08.06.006 